Research Paper

\title{
Efficacy of Betal Integrin and EGFR Targeting in Sphere-Forming Human Head and Neck Cancer Cells
}

\author{
Katja Zscheppang $1,2^{*}$, Ina Kurth ${ }^{1,3^{*}}$, Nicole Wachtel ${ }^{1}$, Anna Dubrovska1, ${ }^{1,6,6,7}$, Leoni A. Kunz-Schughart ${ }^{1,5}$, and \\ Nils Cordes $1,4,5,6,7 凶$ \\ 1. OncoRay - National Center for Radiation Research in Oncology, Faculty of Medicine and University Hospital Carl Gustav Carus, Technische Universität Dresden, and \\ Helmholtz-Zentrum Dresden - Rossendorf, Germany; \\ 2. Institute of Radiopharmaceutical Cancer Research, Helmholtz-Zentrum Dresden - Rossendorf, Germany; \\ 3. National Center for Tumor Diseases (NCT), Partner Site Dresden, Germany; \\ Department of Radiation Oncology, University Hospital and Medical Faculty Carl Gustav Carus, Technische Universität Dresden, Germany; \\ Institute of Radiooncology, Helmholtz-Zentrum Dresden - Rossendorf, Dresden, Germany; \\ German Cancer Consortium (DKTK), Dresden, Germany; \\ . German Cancer Research Center (DKFZ), Heidelberg, Germany. \\ * These authors contributed equally to this work.
}

$\triangle$ Corresponding author: Nils Cordes, MD, PhD. OncoRay - National Center for Radiation Research in Oncology, Medizinische Fakultät Carl Gustav Carus, Technische Universität Dresden, Fetscherstrasse 74 / PF 41, 01307 Dresden, Germany. Phone: ++49 (0)351-458-7401 Fax: ++49 (0)351-458-7311 e-mail: Nils.Cordes@OncoRay.de.

(C) Ivyspring International Publisher. Reproduction is permitted for personal, noncommercial use, provided that the article is in whole, unmodified, and properly cited. See http://ivyspring.com/terms for terms and conditions.

Received: 2015.10.26; Accepted: 2016.01.22; Published: 2016.04.02

\begin{abstract}
Background: Resistance to radiotherapy continues to be a limiting factor in the treatment of cancer including head and neck squamous cell carcinoma (HNSCC). Simultaneous targeting of $\beta 1$ integrin and EGFR was shown to have a higher radiosensitizing potential than mono-targeting in the majority of tested HNSCC cancer models. As tumor-initiating cells (TIC) are thought to play a key role for therapy resistance and recurrence and can be enriched in sphere forming conditions, this study investigated the efficacy of $\beta 1$ integrin/EGFR targeting without and in combination with X-ray irradiation on the behavior of sphere-forming cells (SFC).

Methods: HNSCC cell lines (UTSCC15, UTSCC5, Cal33, SAS) were injected subcutaneously into nude mice for tumor up-take and plated for primary and secondary sphere formation under non-adhesive conditions which is thought to reflect the enrichment of SFC and their self-renewal capacity, respectively. Treatment was accomplished by inhibitory antibodies for $\beta 1$ integrin (AllB2) and EGFR (Cetuximab) as well as X-ray irradiation (2-6 Gy single doses). Further, flow cytometry for TIC marker expression and cell cycling as well as Western blotting for DNA repair protein expression and phosphorylation were employed.

Results: We found higher primary and secondary sphere forming capacity of SAS cells relative to other HNSCC cell lines, which was in line with the tumor up-take rates of SAS versus UTSCC15 cells. AllB2 and Cetuximab administration had minor cytotoxic and no radiosensitizing effects on SFC. Intriguingly, secondary SAS spheres, representing the fraction of surviving SFC upon passaging, showed greatly enhanced radiosensitivity compared to primary spheres. Intriguingly, neither AllB2 nor Cetuximab significantly altered basal sphere forming capacity and radiosensitivity. While an increased accumulation of G0/G1 phase cells was observable in secondary SAS spheres, DNA double strand break repair indicated no difference on the basis of significantly enhanced ATM and Chk2 dephosphorylation upon irradiation.

Conclusions: In the HNSCC model, sphere-forming conditions select for cells, which are unsusceptible to both anti- $\beta 1$ integrin and anti-EGFR inhibitory antibodies. With regard to primary and secondary sphere formation, our data suggest that both of these SFC fractions express distinct survival strategies independent from $\beta 1$ integrin and EGFR and that future work is warranted to better understand SFC survival and enrichment before and after treatment to untangle the underlying mechanisms for identifying novel, druggable cancer targets in SFC.
\end{abstract}

Key words: Sphere formation, head and neck squamous cell carcinoma, radiosensitivity, beta1 integrin, EGFR. 


\section{Introduction}

Human head and neck squamous cell carcinoma (HNSCC) is the sixth most common cancer worldwide with a 3-year survival rate of approximately $30-50 \%$ at advanced-stages [1,2]. Importantly and despite multimodal treatment strategies including surgery and radiochemotherapy, the cure rates of this disease are unchanged for almost three decades. This scenario gives rise to the hypothesis that tumor-initiating cells (TIC) play a fundamental role in locoregional tumor control failure, limited patient overall survival rates and tumor relapse [3-6].

Based on the concept of tumor bulk cells being more sensitive to radiotherapy than TIC, an intensive screening was conducted for TIC-specific, druggable targets. With a proven clinical benefit, targeting the epidermal growth factor receptor (EGFR) with Cetuximab significantly increased both locoregional control and overall survival of patients with locally advanced HNSCC when combined with radiotherapy $[7,8]$. However, Cetuximab appears without TIC-specificity and similarly effective than radiochemotherapy with cisplatin ruling out potential and differential actions on bulk cells and TIC $[9,10]$.

Prosurvival bypass signaling could be one factor critically influencing EGFR targeting. Such actions can appear at the cell membrane or in the cytoplasm. We and others demonstrated for anti-EGFR therapeutics like Cetuximab and Erlotinib a reduced efficacy due to increased ErbB2 signaling, EGFR-Src family kinase interactions or activation of JNK2 signaling via interaction of EGFR and JIP-4 [11-13]. Recently, we evidently exhibited that blocking of $\beta 1$ integrin stimulates B-Raf-MEK1/2-Erk1/2 signaling, which can be prevented by simultaneous $\beta 1$ integrin/EGFR inhibition resulting in enhanced radiosensitization in-vitro and complete tumor cure in-vivo [14]. Thus, targeting of $\beta 1$ integrins has a strong potential to sensitize cancer cells to conventional radio- and chemotherapies $[15,16]$. In general, $\beta 1$ integrins mediate radiochemoresistance via the FAK-Cortactin-JNK1 signaling axis [17]. Interestingly, this integrin associated cascade critically influences the non-homologous endjoining (NHEJ) DNA repair process as well as the distribution of eu- and heterochromatic DNA double strand breaks (DSB) $[18,19]$. Ataxia telangiectasia mutated (ATM), Ku70, $\mathrm{Ku} 80$ and Chk2 are some of the key determinants of the DNA damage response while heterochromatin protein $1 \alpha(\mathrm{HP} 1 \mathrm{a})$ and histone $\mathrm{H} 3$ acetylation are indicators of the chromatin organization [20,21].

The question we addressed here is how SFC of different HNSCC cell lines react to simultaneous $\beta 1$
integrin/EGFR targeting in combination with radiotherapy and how these HNSCC TIC behave when repeatedly plated under non-adherent sphere forming TIC culture conditions. We used the concept of primary and secondary spheres which is thought to enrich for treatment resistant SFC as anticipated for the hypothesis of cancer stem cell repopulation under radiotherapy [22]. The susceptibility neither to a simultaneous $\quad \beta 1$ integrin/EGFR inhibition plus/minus irradiation nor to irradiation alone of these two cell subpopulations has been elucidated.

\section{Materials and Methods}

\section{Antibodies}

Antibodies against FAK, $\beta 1$ Integrin, EGFR, p44/42 MAPK, p-p44/42 MAPK T202/Y204, ATM, Ku80, Chk2, pChk2 T68, Histone H3, HP1a (Cell Signaling), $\beta$-Actin (Sigma), Ku70, Oct4 (Abcam), p-ATM S1981 (Rockland), acetyl-Histone H3 (Millipore), 5-Bromo-2'-deoxyuridine (BrdU)-FITC (BD), HRP-conjugated donkey anti-rabbit and sheep anti-mouse secondary antibodies (GE Healthcare), Alexa Fluor488, Alexa Fluor594, p-EGFR Y1068 (Invitrogen), 53BP1 (Novus Biologicals) were purchased as indicated. The $\beta 1$ integrin inhibitory antibody, clone AIIB2, was isolated from a human choriocarcinoma hybridoma and is a rat monoclonal IgG1 [17]. EGFR blocking was achieved with the antibody Erbitux ${ }^{\circledR}$ (Cetuximab; Merck).

\section{In vivo tumorigenicity experiments}

NMRI (nu/nu) mice were used (pathogen-free breeding facility, Experimental Center, Medical Faculty, Technical University, Dresden, Germany) for subcutaneous injection of UTSCC15 and SAS cells. The animal facilities and the experiments were approved in accordance with institutional guidelines and the German animal welfare regulations (ethical approval reference number: 24D-9168.11-1/2010-21). For further immunosuppression, animals were whole body irradiated with 4 Gy (200 kV x-rays, $0.5 \mathrm{~mm}$ Cu-filter, $\sim 1$ Gy/ min) 3 days before cell injection. Cells were cultured under 2D cell culture conditions in DMEM supplemented with $10 \%$ fetal calf serum and $1 \%$ non-essential amino acids or under 3D cell culture conditions embedded in a laminin-rich extracellular matrix (lrECM (Matrigel ${ }^{\mathrm{TM}}$ ); $\mathrm{BD}$ ) as published $[18,23]$. For tumor development, different cell numbers were injected subcutaneously into the left hind-leg of the mice in $60 \mu \mathrm{L}$ of $\mathrm{BD}$ matrigel (UTSCC15: 10, 102, 103, $10^{4}$ cells; SAS: 12, 25, 102, $10^{3}$ 
cells). Four mice were used for each condition. The tumors were measured every 4 to 5 days and the mice were observed for 5 months for the development of tumors.

\section{Cell cultures and radiation exposure}

Human squamous cell carcinoma cell lines (UTSCC15, UTSCC5, Cal33 and SAS) of the head and neck (HNSCC) were kindly provided by R. Grenman (Turku University Central Hospital, Turku, Finland). Cells were cultured in Dulbecco's Modified Eagle Medium (PAA; plus glutamax-I) supplemented with $10 \%$ fetal calf serum (Biochrom) and 1\% non-essential amino acids (PAA) at $37^{\circ} \mathrm{C}$ in a humidified atmosphere containing $7 \% \quad \mathrm{CO}_{2}$. Irradiation was applied at room temperature using single doses of 200 $\mathrm{kV}$ x-rays (Yxlon Y.TU320; Yxlon) filtered with 0.5 $\mathrm{mm} \mathrm{Cu}$. The absorbed dose was measured using a Duplex Dosimeter (PTW). The dose-rate was approximately $1.3 \mathrm{~Gy} / \mathrm{min}$ at $20 \mathrm{~mA}$ and the applied dose ranged from 0 to $6 \mathrm{~Gy}$.

\section{Sphere assay and treatment}

Human squamous cell carcinoma cell lines (UTSCC15, UTSCC5, Cal33 and SAS; 500 cells per well) were cultured in 24 well ultra-low attachment plates (Corning Inc., Corning, NY). Cells were grown in serum-free Epithelial Basal Medium supplemented with $4 \mathrm{mg} / \mathrm{mL}$ insulin, B27 supplement, $20 \mathrm{ng} / \mathrm{mL}$ epidermal growth factor EGF and $20 \mathrm{ng} / \mathrm{mL}$ basic fibroblast growth factor bFGF. Cells were treated with AIIB2 $(10 \mu \mathrm{g} / \mathrm{ml}$ final concentration), Cetuximab (5 $\mu \mathrm{g} / \mathrm{ml}$ final concentration) or AIIB2+Cetuximab (10 $\mu \mathrm{g} / \mathrm{ml}$ plus $5 \mu \mathrm{g} / \mathrm{ml}$, respectively, final concentration) for $24 \mathrm{~h}$ prior to irradiation with 2, 4 or 6 Gy single $\mathrm{x}$-ray doses. Non-specific IgG isotype antibodies were used as control (10 $\mu \mathrm{g} / \mathrm{ml}$ final concentration). Spheres, defined as non-adherent spheres of $\geq 25$ cells, were imaged and microscopically counted after 8 days. To investigate the formation of secondary spheres from the surviving cells of the first sphere forming assay, spheres were trypsinized for harvesting a single cell suspension. These single cells were plated a second time in 24 well ultra-low attachment plates with serum-free Epithelial Basal Medium. After $24 \mathrm{~h}$, cells were treated and irradiated (2-6 Gy) and secondary spheres were counted 8 days later.

\section{Total protein extracts and Western Blotting}

UTSCC15 and SAS cells were cultured under sphere-forming conditions in $10 \mathrm{~cm}$ ultra-low attachment dishes in serum-free Epithelial Basal Medium. Again, we plated cells for the primary and the secondary sphere formation. Primary plating: Twenty-four hours after plating, cells were treated with AIIB2, Cetuximab or both, as mentioned above, and proteins were harvested from surviving spheres 8 days later using modified RIPA buffer $(50 \mathrm{mM}$ Tris- $\mathrm{HCl}$ ( $\mathrm{pH} 7.4$ ), 1\% Nonidet-P40, $0.25 \%$ sodium deoxycholate, $150 \mathrm{mM} \mathrm{NaCl}, 1 \mathrm{mM}$ EDTA, Complete protease inhibitor cocktail, $1 \mathrm{mM} \mathrm{NaVO}_{4}, 2 \mathrm{mM} \mathrm{NaF}$ ). Secondary plating: Untreated primary spheres were trypsinized for single cell suspension and plated for a second time. Cells were exposed to AIIB2, Cetuximab and the combination as described and proteins were harvested 8 days later. For investigating DNA repair proteins, cells were irradiated with 6 Gy and whole cell lysates were obtained $30 \mathrm{~min}, 2 \mathrm{~h}$ and $24 \mathrm{~h}$ later. After 8 days in culture unirradiated cells were reseeded and secondary spheres were irradiated with 6 Gy or left unirradiated and whole cell lysates were harvested after $30 \mathrm{~min}, 2 \mathrm{~h}$ and $24 \mathrm{~h}$. Lysates were subjected to SDS-PAGE and Western blotting as described [18]. Probing and detection of specific proteins were performed with indicated antibodies.

\section{Flow cytometric analysis of EGFR, $\beta 1$ Integrin and ALDH}

UTSCC15 and SAS cells were cultured under sphere-forming conditions in $10 \mathrm{~cm}$ ultra-low attachment dishes in serum-free Epithelial Basal Medium and EGFR and $\beta 1$ integrin expression was assessed after $24 \mathrm{~h}$ and 8 days. Briefly, single cells were collected as described above and incubated with specific anti-EGFR or anti- $\beta 1$ integrin antibodies for 30 minutes, washed three times and incubated with secondary antibodies for 30 minutes. For determination of ALDH activity, SAS cells were stained with ALDFLUOR ${ }^{\text {TM }}$ (Stemcell Technologies) according to the manufacturer's instructions. Treatment of cells with diethylaminobenzaldehyde (DEAB; specific inhibitor of ALDH activity) served as control. Propidium iodide (PI) staining was used for elimination of dead cells and cells were sorted on FACSAriaII (Becton Dickinson). The percentage of positive cells was determined by Overton subtraction of the fluorescence profiles of the positive and isotype control cell populations.

\section{Cell cycle analysis}

SAS cells were cultured under sphere-forming conditions for $24 \mathrm{~h}$ as described above. Before harvesting, cells were incubated with $10 \mathrm{mM}$ BrdU and then prepared for cell cycle measurement as published [15]. In brief, cells were trypsinized, washed with PBS and fixed in $80 \%$ ethanol until use. Then, cells were prepared for analysis by incubation with RNase A and $\mathrm{HCl}$ solutions. Subsequently, probing BrdU with mouse anti-BrdU antibodies and total DNA staining with PI was accomplished. Cell 
cycle was determined by flow cytometry.

\section{Detection of DNA double strand breaks}

For detection of residual DNA double strand breaks (DSB), p53 binding protein-1 (p53BP1) was stained [18]. UTSCC15 and SAS cells were cultured under sphere-forming conditions. After $24 \mathrm{~h}$, cells were irradiated with 6 Gy or left unirradiated. Two and $24 \mathrm{~h}$ later, cells were isolated for fixation $(3 \%$ formaldehyde/PBS) and permeabilization (0.25\% Triton X-100/PBS) using trypsin. After blocking with $1 \%$ BSA, cells were stained with a specific anti-p53BP1 antibody and embedded in Vectashield/DAPI mounting medium. $\mathrm{p} 53 \mathrm{BP} 1$ positive nuclear foci were determined in at least 150 cells using an Axioscope 2 plus fluorescence microscope (Zeiss, Jena, Germany).

\section{Statistical analysis}

Data are presented as mean \pm SD of at least 3 independent experiments. The level of significance was determined by unpaired, 2-sided Student's t-test using Microsoft Excel. Results were considered statistically significant if a $P$-value of less than 0.05 was reached.

\section{Results}

\section{HNSCC SFC show basal Cetuximab/AIIB2 susceptibility but no radiosensitization}

Our previous work identified responder and non-responder HNSCC tumor models for simultaneous $\beta 1$ integrin/EGFR targeting [14]. As responder model, we used UTSCC15 and as non-responder model SAS. We tested the cytotoxic and radiosensitizing potential of Cetuximab and the anti- $\beta 1$ integrin antibody AIIB2 on SFC from UTSCC15 and SAS cell lines (Figure 1A). To elucidate changes in the susceptibility of these SFC to the chosen therapy over time, primary spheres were harvested, dissociated, and single cells were plated for secondary sphere formation (Figure 1A). The monotherapies and also the combined treatment of AIIB2 and Cetuximab caused a significant cytotoxicity in both HNSCC SFC as determined by the number of evolving spheres 8 days after treatment (Figure 1B, left panel) but had no impact on radiosensitivity (Figure 1C, left panel).

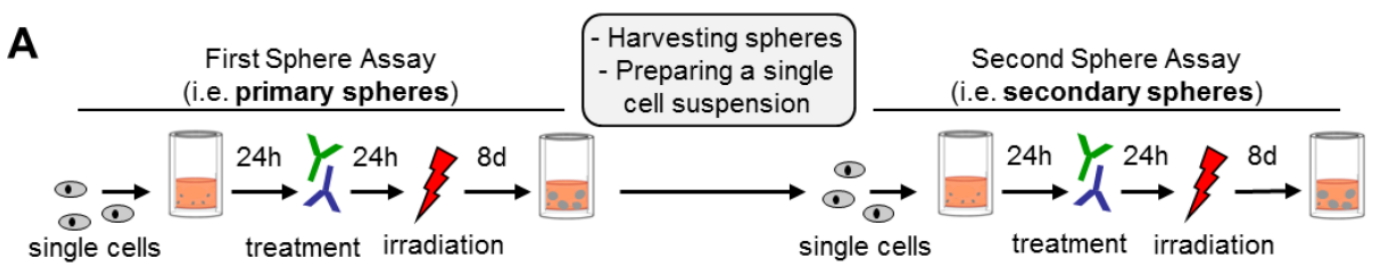

B
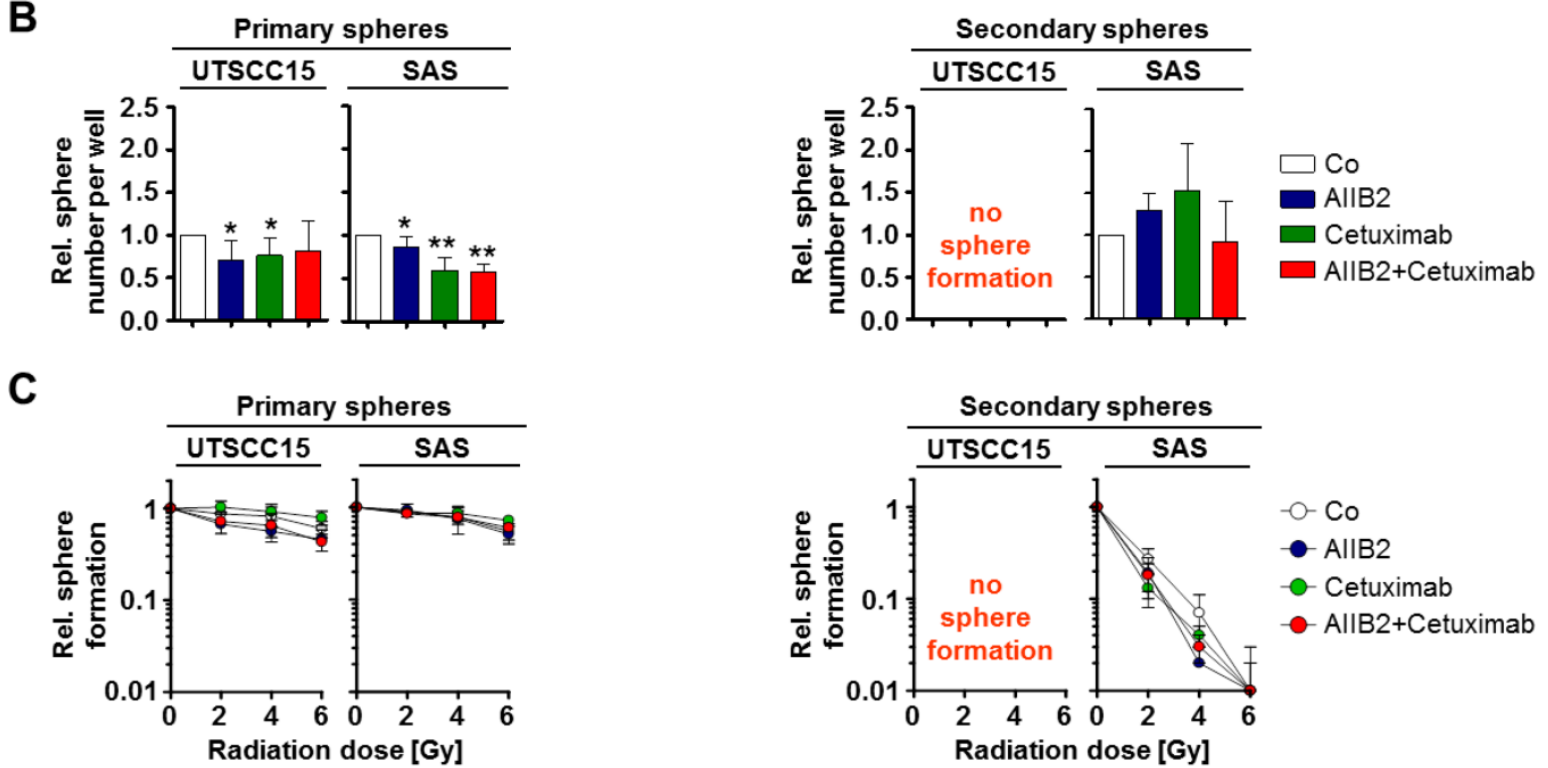

Figure 1. Primary and secondary HNSCC cell spheres show different treatment sensitivity. (A) Treatment scheme. Cells were plated under non-adherent conditions in serum-free Epithelial Basal Medium for sphere formation. After 8 days, spheres of untreated cells were harvested and plated for secondary sphere formation. (B) Number of primary and secondary spheres after AllB2, Cetuximab and combined AllB2/Cetuximab (IgG as control, Co). (C) Number of primary and secondary spheres upon irradiation (2-6 Gy) with and without AlIB2 and Cetuximab treatment. Results show mean \pm SD $(n=3 ; t$-test; * $P<0.05, * * P<0.01)$. 
Upon re-seeding of SFC for secondary sphere formation, to test their self-renewal capacity, we exhibited no sphere formation for UTSCC15 cells (Figure 1A and B, right panel) and an abolished Cetuximab/AIIB2 susceptibility in SAS (Figure 1B, right panel). Intriguingly, the radiosensitivity of SAS cells plated for secondary sphere formation was dramatically increased and independent from AIIB2 or Cetuximab co-treatment (Figure $1 \mathrm{~B}$ and $\mathrm{C}$, right panel).

These findings suggest HNSCC SFC growing at non-adherent conditions to be resistant to single and double targeting strategies for $\beta 1$ integrin and EGFR. HNSCC cells from primary sphere culture conditions show a highly differential capability to form secondary spheres and survive exposure to ionizing radiation.

\section{SAS cells show a higher tumorigenicity in mice than UTSCC15 cells}

Next, we evaluated the tumor take rate of the responder model, i.e. UTSCC15, versus the non-responder model, i.e. SAS. Cells of these cell lines were subcutaneously injected into nude mice and assessed for their tumor formation over 5 month. Before injection, the cells were cultured either under 2D monolayer conditions or under non-adherent sphere forming conditions. As a result, we found the tumorigenicity of the non-responder SAS cells to be higher than of the responder UTSCC15 cells independent from the growth conditions before injection (Table 1). Our observations indicate a yet to be identified molecular phenotype that elicits a clear survival advantage in the HNSCC non-responder cell line SAS relative to the responder cell line UTSCC15.

Table 1. Tumorigenicity of UTSCC15 and SAS cells.

\begin{tabular}{lllllllll}
\hline & UTSCC15 & \multicolumn{7}{c}{ SAS } \\
\hline Cells injected & $\mathbf{1 0}$ & $\mathbf{1 0 0}$ & $\mathbf{1 0 0 0}$ & $\mathbf{1 0 0 0 0}$ & $\mathbf{1 0}$ & $\mathbf{2 5}$ & $\mathbf{1 0 0}$ & $\mathbf{1 0 0 0}$ \\
2D monolayer & $0 / 4$ & $1 / 4$ & $3 / 4$ & $4 / 4$ & $4 / 4$ & $4 / 4$ & $4 / 4$ & $4 / 4$ \\
Spheres & $0 / 4$ & $0 / 4$ & $0 / 4$ & $0 / 4$ & $0 / 4$ & $0 / 4$ & $0 / 4$ & $1 / 4$ \\
\hline
\end{tabular}

Numbers indicate number of animals showing tumors/number of animals receiving tumor cells.

\section{$\beta 1$ integrin and EGFR target expression and signal transduction}

To test whether the responder UTSCC15 and non-responder SAS HNSCC cells are characterized by distinct $\beta 1$ integrin and EGFR surface expression under sphere formation conditions, we determined the $\beta 1$ integrin and EGFR cell surface expression status in untreated spheres $24 \mathrm{~h}$ and 8 days after seeding (Figure 2A). Upon treatment with AIIB2 and/or Cetuximab, we further measured the expression and phosphorylation of associated downstream protein targets in cells grown under sphere forming conditions (Figure 2-B, C). While the positive $\beta 1$ integrin and EGFR cell surface expression in UTSCC15 sphere forming cells was stable between day 1 and 8 (Figure 2A), a strong increase in the percentage of SAS cells with receptor positivity was detectable between day 1 and 8 in the primary sphere culture (Figure 2A).
A
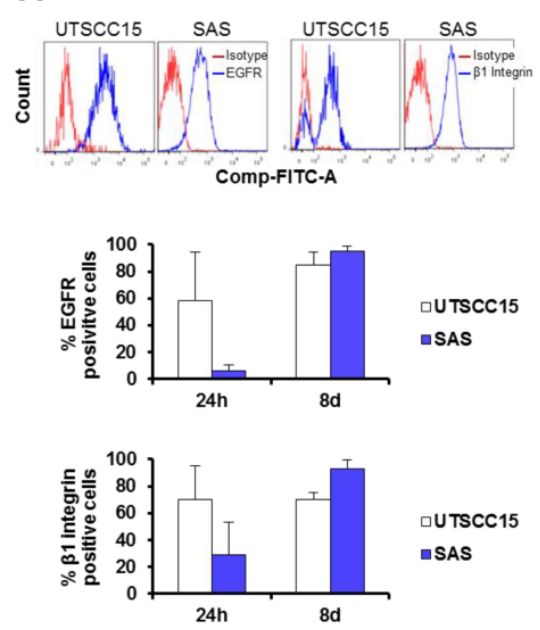

B

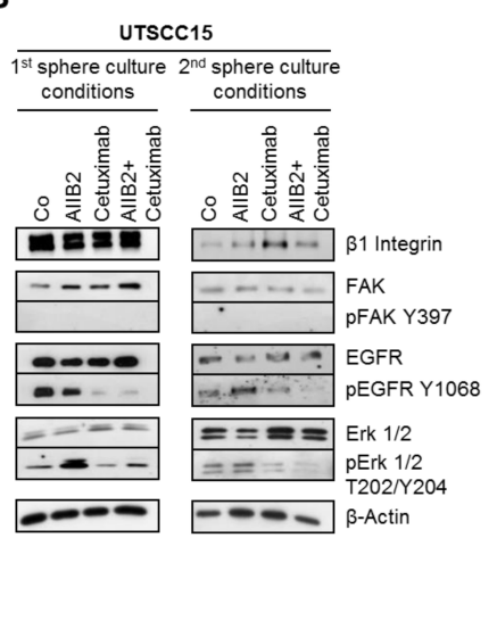

C

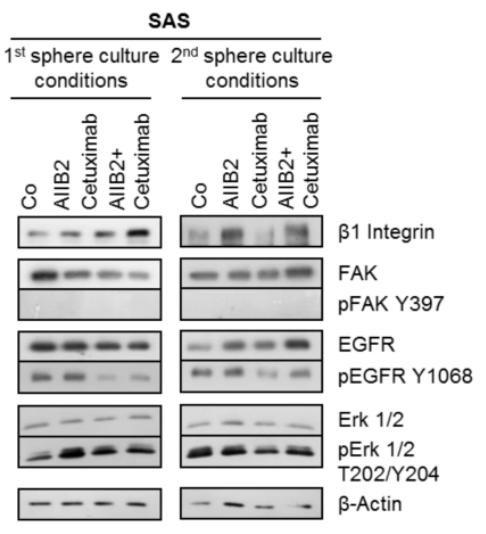

Figure 2. $\beta 1$ integrin and EGFR expression are similar in primary and secondary spheres. (A) EGFR and $\beta 1$ integrin surface expression in UTSCC15 and SAS primary spheres analyzed by flow cytometry. (B) Western blot analysis of indicated proteins on whole cell lysates from UTSCC15 and SAS primary and (C) secondary spheres. $\beta$-Actin served as loading control. Results show mean \pm SD $(n=3)$. 
On the level of whole protein cell lysate analysis 8 days after primary or secondary sphere culture conditions with or without treatment, we found reduced EGFR Tyrosine (Y) 1068 phosphorylation in Cetuximab-treated UTSCC15 and SAS cells (Figure 2B and C). Interestingly, AIIB2 induced Erk1/2 Threonine (T) 202/Y204 phosphorylation in primary spheres of both cell lines. However, upon Cetuximab treatment Erk1/2 dephosphorylation was only observed in UTSCC15 responder in contrast to SAS non-responder cells. Due to non-adherent growth conditions, FAK was completely dephosphorylated at amino acid residue Y397 in both cell lines (Figure 2B and $\mathrm{C}$ ).

Similar to our investigations in HNSCC grown in laminin-rich extracellular matrix [14], the $\beta 1$ integrin and EGFR cell surface patterns do not predict for outcome without or with radiotherapy.

\section{Sphere forming capacities vary between $\boldsymbol{\beta} 1$ integrin/EGFR responder and non-responder HNSCC cell lines}

Subsequently, we examined the primary and secondary sphere forming capacity of additional HNSCC cell lines, i.e. UTSCC5, Cal33, previously characterized as $\beta 1$ integrin/EGFR responder cell

A

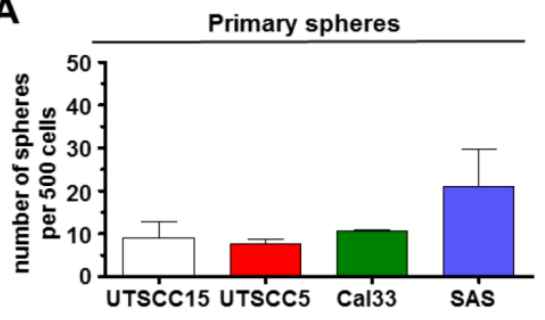

C
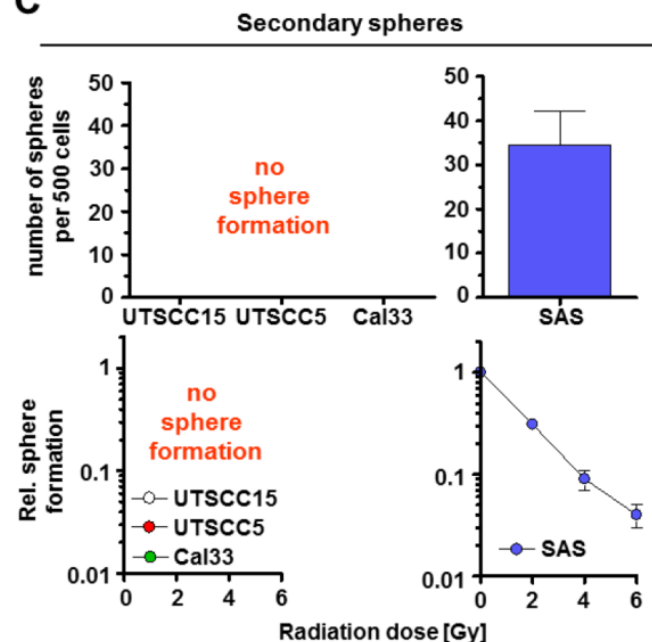

Figure 3. HNSCC cell lines exhibit different capacity for secondary sphere formation. Number of spheres formed per 500 non-irradiated (A) and irradiated (2 - 6 Gy) (B) cells. (C) Number of secondary spheres per 500 cells and sphere formation (2 - 6 Gy) after irradiation of unirradiated secondary spheres. Results represent mean $\pm S D(n=3)$. lines according to UTSCC15 [14]. Amongst these cell lines, the non-responder SAS cells demonstrated the highest primary spheres forming capacity (Figure $3 \mathrm{~A})$. Upon irradiation, primary sphere growth revealed marginal differences between the responder cell lines UTSCC15, UTSCC5 and Cal33 as compared to the non-responder cell line SAS (Figure 3B). Similar to UTSCC15 cells, UTSCC5 and Cal33 cells were incapable of forming secondary spheres (Figure 3C). As described above, SAS cell radiosensitivity was dramatically enhanced under secondary sphere forming conditions (Figure 3C) relative to primary spheres (Figure 3B). SAS indicate to express a specific molecular phenotype that mediates a survival advantage relative to other HNSCC cell lines.

\section{Sphere formation of ALDH positive and negative cell populations}

The following experiments were performed to gain insight into the phenomenon of enhanced susceptibility of SAS in secondary spheres to irradiation and the interrelation with putative, functional cancer stem cell markers and DNA repair signaling. First, SAS cells were sorted for high and low ALDH activity as a marker for SFC in HNSCC [24-26] (Figure 4A). Subsequent plating under non-adherent conditions for sphere formation revealed that only ALDH positive cells are able to form spheres (Figure 4A). A comparative analysis of ALDH activity showed that primary SAS spheres consist of a higher number of ALDH positive cells than secondary spheres (Figure 4B). Additionally, we determined the expression of Oct4 as a marker for self-renewal and undifferentiated cells $[27,28]$ and found a significantly higher Oct4 expression in secondary relative to primary spheres (Figure $4 \mathrm{C}$ ).

Moreover, our study on cell cycle distribution indicated an enrichment of cells in the G0/G1 phase in favor of cells in S phase when comparing secondary versus primary spheres (Figure 5A).

To find an explanation for the high radiosensitivity of secondary relative to primary spheres, the DSB number was measured at $2 \mathrm{~h}$ and $24 \mathrm{~h}$ after irradiation. Interestingly, we were unable to detect any difference in the repair of radiogenic 53BP1 positive foci between primary and the secondary spheres (Figure 5B).

These findings indicate differential SFC marker expression and cell cycle distribution in primary and secondary spheres while the DNA repair of radiogenic DNA double strand breaks is similar. 
DNA repair signaling in primary and secondary HNSCC spheres

Despite of the aforementioned similarity in DSB repair, the markedly enhanced radiosensitivity of the secondary relative to primary spheres motivated us to investigate the expression of DNA repair proteins. When comparing basal expression and phosphorylation levels, cells form secondary spheres showed significantly reduced Chk2 T68 phosphorylation and histone $\mathrm{H} 3$ acetylation than those from primary spheres (Figure 6A and B). This was paralleled by increased HP1a expression. Other proteins of interest remained stable in their expression.

Upon 6-Gy irradiation, we detected a significantly accelerated ATM Serine (S) 1981 and a strong Chk2 T68 dephosphorylation in secondary relative to primary spheres (Figure 6A and B). Ku70, Ku80, HP1a and histone H3 acetylation showed no significant differences in irradiated primary and secondary spheres (Figure 6A and B).

In contrast to the data from the foci assay (Figure 5B), ATM and Chk2 seem perturbed in secondary spheres, which may in part explain the high radiosensitivity of this cell population.
A
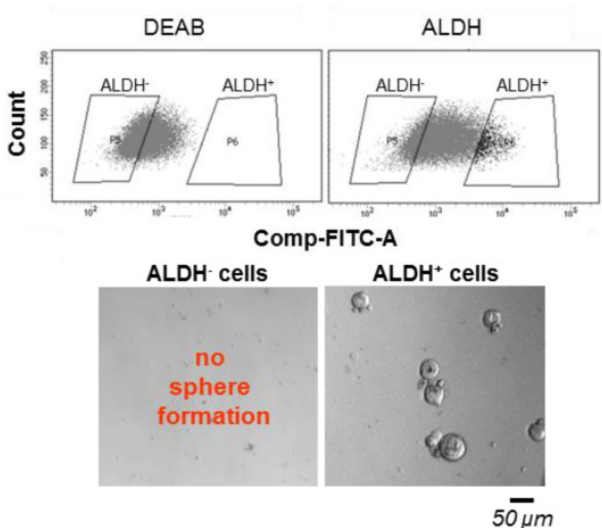

B
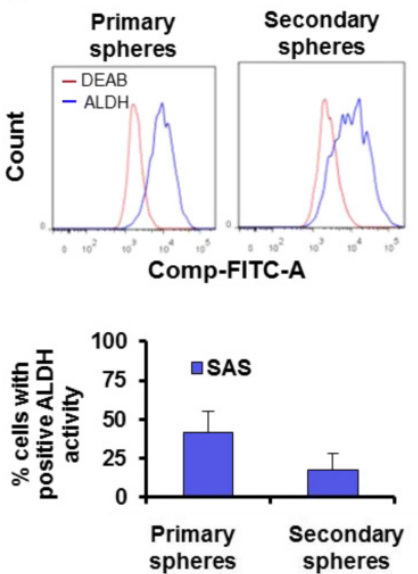

C

Primary Secondary spheres spheres
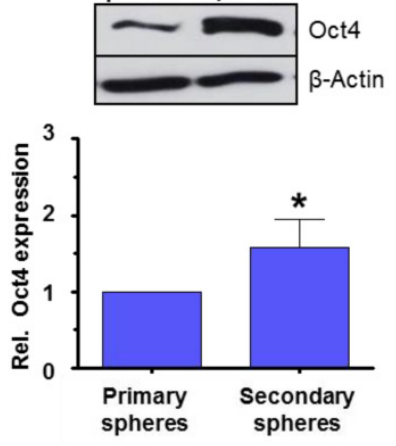

Figure 4. Role of ALDH activity as a HNSCC TIC marker in sphere formation. (A) SAS cells sorted for ALDH positivity and plated for sphere formation. (B) Analysis of ALDH activity by flow cytometry and (C) Oct4 expression by Western blotting $24 \mathrm{~h}$ after the start of the primary sphere culture and $24 \mathrm{~h}$ after the start of the secondary sphere culture. Results show mean \pm SD $(n=3$; t-test; $* P<0.05)$.

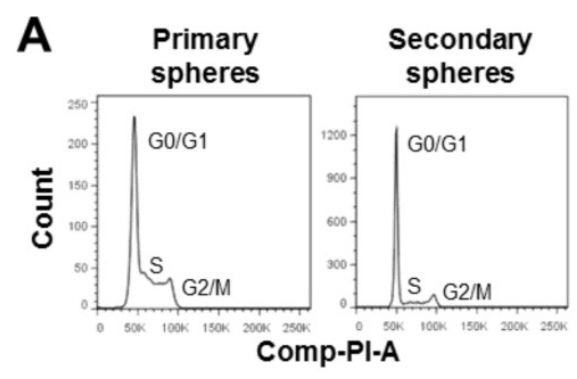

B

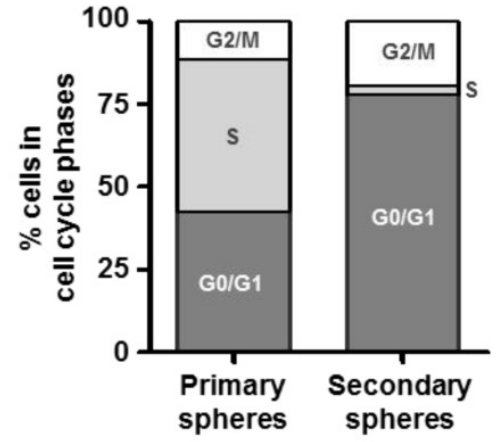

Secondary spheres

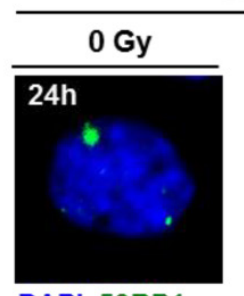

DAPI, 53BP1

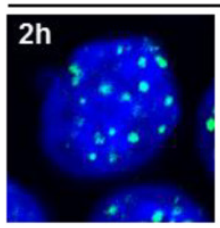

24h
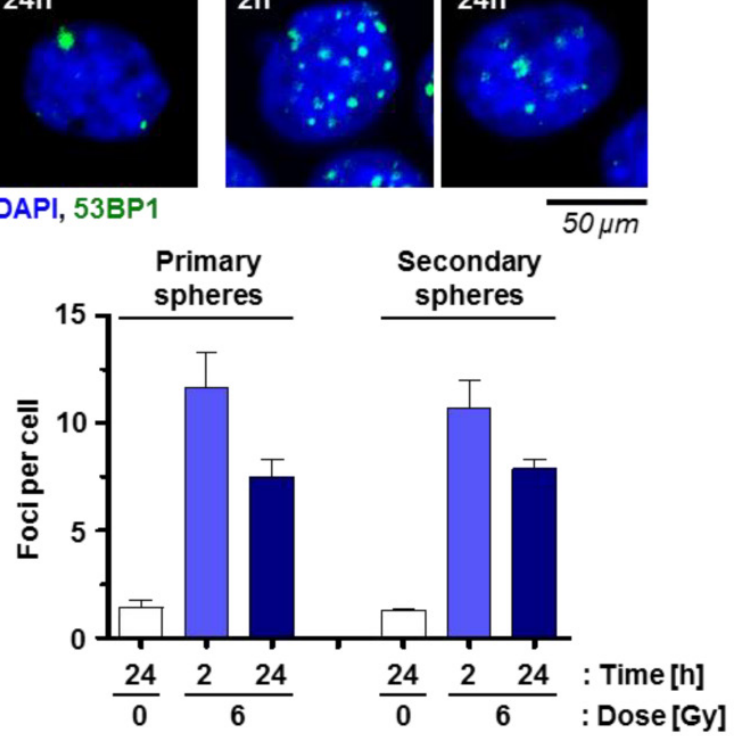

Figure 5. Cell cycling but not DSB repair varies between primary and secondary spheres of SAS cells. (A) Flow cytometric cell cycle analysis of primary and secondary spheres. (B) Quantification of p53BPI positive radiation-induced foci per cell $2 \mathrm{~h}$ and $24 \mathrm{~h}$ after 6 -Gy $\mathrm{X}$-rays irradiation. Results show mean \pm SD ( $\mathrm{n}=3$ ). 
A

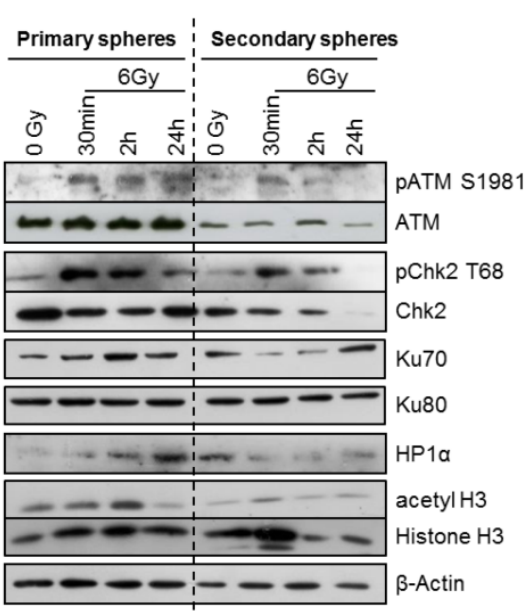

B

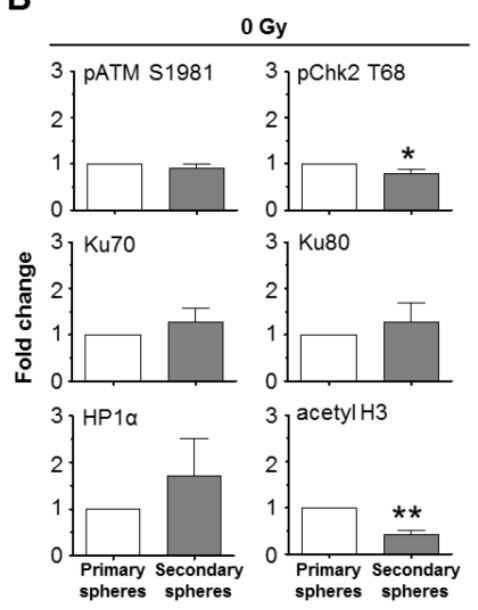

C

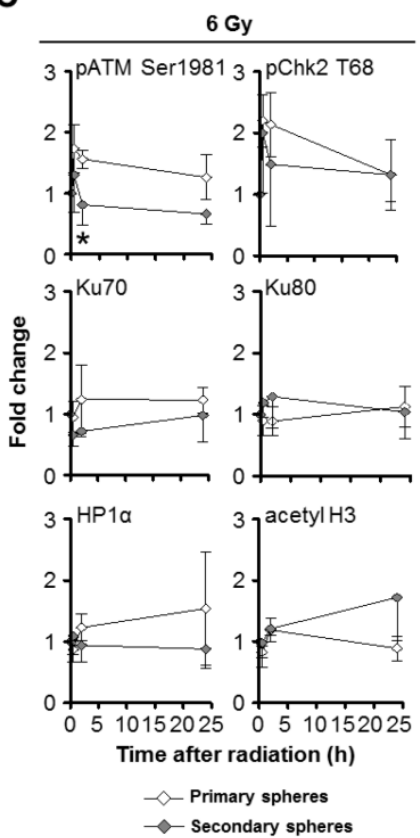

Figure 6. DNA repair and chromatin marker proteins are differentially expressed and phosphorylated in primary and secondary SAS spheres. Western blot (A) and corresponding densitometric analysis of unirradiated (B) and irradiated (C) SAS primary and secondary sphere cultures. $\beta$-Actin served as loading control. Results show mean $\pm S D(n=3$; t-test; $* P<0.05$, ** $P<0.01)$.

\section{Discussion}

Resistance to cancer therapy continues to be a limiting factor in the treatment of cancer including HNSCC. Simultaneous targeting of $\beta 1$ integrin and EGFR was shown to have a higher radiosensitizing potential than mono-targeting in the majority of tested HNSCC cancer models [14]. TIC markers were linked previously to poor outcome in HNSCC patients, with $\beta 1$ integrin as one of the strongest and independent prognostic TIC factor [22]. As TIC are thought to play a key role for therapy resistance, this study investigated the efficacy of $\beta 1$ integrin/EGFR targeting without and in combination with X-ray irradiation on SFC behavior. We found that (i) SAS cells, i.e. non-responder to AIIB2, show a higher tumorigenicity in mice than AIIB2 responder UTSCC15 cells, (ii) HNSCC TIC show basal Cetuximab/AIIB2 susceptibility but no radiosensitization, (iii) $\beta 1$ integrin and EGFR were differentially expressed on UTSCC15 and SAS cells including an AIIB2-induced Erk1/2 phosphorylation in primary but not secondary spheres of both cell lines, (iv) sphere forming capacities vary between $\beta 1$ integrin/EGFR responder and non-responder HNSCC cell lines, (v) sphere formation was only observed for ALDH positive SAS cell populations, and (vi) DNA repair signaling is compromised in secondary relative to primary SAS spheres.

Cancer cells grown in stem cell media and under low-attachment conditions are thought to have properties of TIC with slow proliferation rate and high therapy resistance [29-34]. Interesting for us was that particularly SAS cells form tumors in nude mice when growing under 2D monolayer cell culture and not under sphere forming conditions. This feature is in line with the absent susceptibility to simultaneous $\beta 1$ integrin/EGFR targeting relative to the responder cell lines UTSCC15, UTSCC5 and Cal33 [14]. Further, serial passaging of cells harvested from spheres in vitro or serial transplantation of HNSCC cells in vivo was described to enrich the malignant subpopulation and the secondary spheres are shown to maintain marker expression specific for the resistant cells and their self-renewing capacity $[30,35,36]$. However, no data are available on the influence of irradiation on passaged spheres.

Comparing sphere forming capacity upon irradiation alone versus irradiation plus antibodies, we surprisingly found that secondary SAS spheres show enhanced radiosensitivity and sphere formation upon Cetuximab. In contrast, UTSCC15, UTSCC5 and Cal33 lost their potential to grow under sphere forming conditions when re-passaged. Our efforts in identifying the underlying mechanisms showed that the two targets, i.e. $\beta 1$ integrin and EGFR, are present on almost $100 \%$ of UTSCC15 and SAS cells before re-passaging and that changes in the major signaling mediators downstream of these two receptors cannot explain the differences in secondary sphere formation upon antibody treatment and irradiation. Interestingly, the non-responder SAS model showed a 
low percentage of $\beta 1$ integrin and EGFR surface expressing cells at the 24-h time point of antibody treatment in contrast to the responder UTSCC15 cells. This observation might, at least partially, explain the difference in treatment susceptibility and warrants identification of the molecular phenotype of non-responder SAS cells as compared with HNSCC responder cell lines.

As quiescence or slow cell cycling might play an important role for therapy sensitivity and repair of DNA damage [37-39], we analyzed the cell cycle distribution in primary and secondary SAS spheres. While secondary spheres demonstrated enrichment of G0/G1-phase cells relative to primary spheres indicative of slow cell cycling, the removal rate of radiation-induced, 53BP1-positive foci showed to be similar in primary and secondary spheres. Generally, it is thought that an elongated cell cycle provides more time for repairing DNA damages via nonhomologous end-joining (NHEJ). This process is error-prone compared to the homologous recombination repair because a template for recombination is unavailable [5]. Since analysis of DNA breaks after irradiation revealed no difference between primary and secondary spheres, we had a closer look on proteins involved in the DNA repair processes. The initial step of a cascade resulting in cell cycle arrest, DNA repair or apoptosis is DSB recognition and ATM activation [40] upon ATM phosphorylation and checkpoint protein activation [41,42]. In our hands, irradiation led to an ATM hyperphosphorylation after 30 minutes. In primary spheres this hyperphosphorylation was maintained over $24 \mathrm{~h}$ whereas in the secondary spheres it was already reduced to baseline levels $2 \mathrm{~h}$ after irradiation. A similar phosphorylation pattern could be observed for the ATM downstream cell cycle checkpoint kinase 2 (Chk2) with regard to its threonine (T) 68 phosphorylation [41,43]. Another important component influencing the DNA damage response and radiation survival is chromatin organization $[18,44-46]$. In line with the quiescence in secondary sphere cells, we found higher HP1a expression levels in secondary than in primary spheres. In contrast, HP1a expression level increased in primary but decreased in secondary spheres upon irradiation. This suggests a different chromatin remodeling mechanism in secondary spheres and therefore a distinct accessibility for the DNA repair machinery. In accordance with increased quiescence and HP1a expression dynamic, histone $\mathrm{H} 3$ acetylation was inversely detectable with decreased protein expression level in secondary spheres. Whether these findings are causative for the differences in radiosensitivity seen in primary versus secondary SAS spheres require further study.

In summary, this study provides a possible explanation for the discrimination between responder and non-responder HNSCC tumor models for $\beta 1$ integrin/EGFR targeted therapy. Quantitative differences in SFC in the various HNSCC models used may relate to the different treatment susceptibility. Further, the SAS spheres analyzed here show some typical characteristics of TIC with therapy resistance and slow cell cycle progression. Future work will elucidate the molecular phenotype and the mechanistic link between chromatin and DNA repair for TIC radiosensitivity in more depth.

\section{Acknowledgements}

We thank M. Huether for excellent technical assistance. All authors discussed the results, commented on the manuscript and approved the final manuscript. All authors conceived and designed the experiments and wrote the manuscript. K.Z., I.K. and N.W. performed the experiments and analyzed the data. The study sponsors did not have any role in the design of the study, the collection, analysis or interpretation of the data, the writing of the article, or the decision to submit the article for publication.

\section{Funding}

The work was in part supported by a grant from the Bundesministerium für Bildung und Forschung (BMBF Contracts 03ZIK041 and BMBF-02NUK006B to N.C.), the EFRE Europäische Fonds für regionale Entwicklung, Europa fördert Sachsen (100066308) and the Helmholtz Virtual Institute NanoTracking (Agreement Number VH-VI-421).

\section{Conflict of interest}

The authors declare that no conflict of interest exists.

\section{References}

[1] Parkin DM, Bray F, Ferlay J, Pisani P. Global cancer statistics, 2002. CA Cancer J Clin 2005;55:74-108.

[2] Siegel R, Naishadham D, Jemal A. Cancer Statistics. CA: A Cancer J Clin 2013 2013;63:11-30. doi:10.3322/caac.21166

[3] Prince ME, Sivanandan R, Kaczorowski a, Wolf GT, Kaplan MJ, Dalerba P, et al. Identification of a subpopulation of cells with cancer stem cell properties in head and neck squamous cell carcinoma. Proc Natl Acad Sci U S A 2007;104:973-8. doi:10.1073/pnas.0610117104.

[4] Peitzsch C, Kurth I, Kunz-Schughart L, Baumann M, Dubrovska A. Discovery of the cancer stem cell related determinants of radioresistance. Radiother Oncol 2013;108:378-87. doi:10.1016/j.radonc.2013.06.003.

[5] Maugeri-Saccà M, Bartucci M, De Maria R. DNA damage repair pathways in cancer stem cells. Mol Cancer Ther 2012;11:1627-36. doi:10.1158/1535-7163.MCT-11-1040.

[6] Chinn SB, Darr $\mathrm{O}$ a, Peters $\mathrm{RD}$, Prince ME. The role of head and neck squamous cell carcinoma cancer stem cells in tumorigenesis, metastasis, and treatment failure. Front Endocrinol (Lausanne) 2012;3:90. doi:10.3389/fendo.2012.00090.

[7] Bonner JA, Harari PM, Giralt J, Azarnia N, Shin DM, Cohen RB, et al. Radiotherapy plus cetuximab for squamous-cell carcinoma of the head and neck. N Engl J Med 2006;354:567-78. doi:10.1056/NEJMoa053422. 
[8] Bonner JA, Harari PM, Giralt J, Cohen RB, Jones CU, Sur RK, et al. Radiotherapy plus cetuximab for locoregionally advanced head and neck cancer: 5-year survival data from a phase 3 randomised trial, and relation between cetuximab-induced rash and survival. Lancet Oncol 2010;11:21-8. doi:10.1016/S1470-2045(09)70311-0.

[9] Fan C-W, Chen T, Shang Y-N, Gu Y-Z, Zhang S-L, Lu R, et al. Cancer-initiating cells derived from human rectal adenocarcinoma tissues carry mesenchymal phenotypes and resist drug therapies. Cell Death Dis 2013;4:e828. doi:10.1038/cddis.2013.337

[10] La Fleur L, Johansson A-C, Roberg K. A CD44high/EGFRlow subpopulation within head and neck cancer cell lines shows an epithelial-mesenchymal transition phenotype and resistance to treatment. PLoS One 2012;7:e44071. doi:10.1371/journal.pone.0044071.

[11] Eke I, Schneider L, Förster C, Zips D, Kunz-Schughart LA, Cordes N. EGFR/JIP-4/JNK2 signaling attenuates cetuximab-mediated radiosensitization of squamous cell carcinoma cells. Cancer Res 2013;73:297-306. doi:10.1158/0008-5472.CAN-12-2021.

[12] Kanda R, Kawahara A, Watari K, Murakami Y, Sonoda K, Maeda M, et al. Erlotinib resistance in lung cancer cells mediated by integrin $\beta 1 /$ Src/Akt-driven bypass signaling. Cancer Res 2013;73:6243-53. doi:10.1158/0008-5472.CAN-12-4502.

[13] Yonesaka K, Zejnullahu K, Okamoto I, Satoh T, Cappuzzo F, Souglakos J, et al. Activation of ERBB2 signaling causes resistance to the EGFR-directed therapeutic antibody cetuximab. Sci Transl Med 2011;3:99ra86. doi:10.1126/scitranslmed.3002442.

[14] Eke I, Zscheppang K, Dickreuter E, Hickmann L, Mazzeo E, Unger K, et al. Simultaneous $\beta 1$ integrin-EGFR Targeting and Radiosensitization of Human Head and Neck Cancer. J Natl Cancer Inst 2015;107. doi:10.1093/jnci/dju419.

[15] Cordes N, Seidler J, Durzok R, Geinitz H, Brakebusch C. beta1-integrin-mediated signaling essentially contributes to cell survival after radiation-induced genotoxic injury. Oncogene 2006;25:1378-90.

[16] Park CC, Zhang HJ, Yao ES, Park CJ, Bissell MJ. Beta1 integrin inhibition dramatically enhances radiotherapy efficacy in human breast cancer xenografts. Cancer Res 2008:68:4398-405. doi:10.1158/0008-5472.CAN-07-6390.

[17] Eke I, Deuse Y, Hehlgans S, Gurtner K, Krause M, Baumann M, et al. $\beta 1$ Integrin/FAK/cortactin signaling is essential for human head and neck cancer resistance to radiotherapy. J Clin Invest 2012;122:1529-40. doi:10.1172/JCI61350.

[18] Storch K, Eke I, Borgmann K, Krause M, Richter C, Becker K, et al. Three-dimensional cell growth confers radioresistance by chromatin density modification. Cancer Res 2010;70:3925-34.

[19] Dickreuter E, Eke I, Krause M, Borgmann K, van Vugt MA, Cordes N. Targeting of $\beta 1$ integrins impairs DNA repair for radiosensitization of head and neck cancer cells. Oncogene 2015. doi:10.1038/onc.2015.212.

[20] Feng FY, de Bono JS, Rubin MA, Knudsen KE. Chromatin to Clinic: The Molecular Rationale for PARP1 Inhibitor Function. Mol Cell 2015;58:925-34. doi:10.1016/j.molcel.2015.04.016.

[21] Dietlein F, Reinhardt HC. Molecular pathways: exploiting tumor-specific molecular defects in DNA repair pathways for precision cancer therapy. Clin Cancer Res 2014;20:5882-7. doi:10.1158/1078-0432.CCR-14-1165.

[22] Koukourakis MI, Giatromanolaki A, Tsakmaki V, Danielidis V, Sivridis E. Cancer stem cell phenotype relates to radio-chemotherapy outcome in locally advanced squamous cell head-neck cancer. Br J Cancer 2012;106:846-53. doi:10.1038/bjc. 2012.33

[23] Eke I, Hehlgans S, Sandfort V, Cordes N. 3D matrix-based cell cultures: Automated analysis of tumor cell survival and proliferation. Int J Oncol 2016;48(1):313-21. doi: 0.3892/ijo.2015.3230.

[24] Kurth I, Hein L, Mäbert K, Peitzsch C, Koi L, Cojoc M, et al. Cancer stem cell related markers of radioresistance in head and neck squamous cell carcinoma. Oncotarget 2015. doi:10.18632/oncotarget.5417.

[25] Clay MR, Tabor M, Owen JH, Carey TE, Bradford CR, Wolf GT, et al Single-marker identification of head and neck squamous cell carcinoma cancer stem cells with aldehyde dehydrogenase. Head Neck 2010;32:1195-201. doi:10.1002/hed.21315.

[26] Chen Y-C, Chen Y-W, Hsu H-S, Tseng L-M, Huang P-I, Lu K-H, et al. Aldehyde dehydrogenase 1 is a putative marker for cancer stem cells in head and neck squamous cancer. Biochem Biophys Res Commun 2009;385:307-13. doi:10.1016/j.bbrc.2009.05.048

[27] Park I-H, Zhao R, West JA, Yabuuchi A, Huo H, Ince TA, et al. Reprogramming of human somatic cells to pluripotency with defined factors. Nature 2008;451:141-6. doi:10.1038/nature06534.

[28] Kumar SM, Liu S, Lu H, Zhang H, Zhang PJ, Gimotty PA, et al. Acquired cancer stem cell phenotypes through Oct4-mediated dedifferentiation. Oncogene 2012;31:4898-911. doi:10.1038/onc.2011.656.

[29] Dontu G, Abdallah WM, Foley JM, Jackson KW, Clarke MF, Kawamura MJ, et al. In vitro propagation and transcriptional profiling of human mammary stem/progenitor cells. Genes Dev 2003;17:1253-70. doi:10.1101/gad.1061803.

[30] Krishnamurthy S, Noer JE. Orosphere assay: A method for propagation of head and neck cancer stem cells. Head Neck 2012:1-7. doi:10.1002/HED.

[31] Alison MR, Lin W-R, Lim SML, Nicholson LJ. Cancer stem cells: in the line of fire. Cancer Treat Rev 2012;38:589-98. doi:10.1016/j.ctrv.2012.03.003.

[32] Tanabe A, Deguchi T, Sato T, Nemoto $Y$, Maruo T, Madarame H, et al. Radioresistance of cancer stem-like cell derived from canine tumours. Vet Comp Oncol 2014. doi:10.1111/vco.12110.
[33] Phillips TM, McBride WH, Pajonk F. The response of CD24(-/low)/CD44+ breast cancer-initiating cells to radiation. J Natl Cancer Inst 2006;98:1777-85. doi:10.1093/jnci/djj495.

[34] Gu D, Liu H, Su GH, Zhang X, Chin-Sinex H, Hanenberg H, et al. Combining hedgehog signaling inhibition with focal irradiation on reduction of pancreatic cancer metastasis. Mol Cancer Ther 2013;12:1038-48. doi:10.1158/1535-7163.MCT-12-1030.

[35] Rybak AP, He L, Kapoor A, Cutz J-C, Tang D. Characterization of sphere-propagating cells with stem-like properties from DU145 prostate cancer cells. Biochim Biophys Acta 2011;1813:683-94. doi:10.1016/j.bbamcr.2011.01.018

[36] Chen C-Y, Chiou S-H, Huang C-Y, Jan C-I, Lin S-C, Tsai M-L, et al. Distinct population of highly malignant cells in a head and neck squamous cell carcinoma cell line established by xenograft model. J Biomed Sci 2009;16:100. doi:10.1186/1423-0127-16-100.

[37] Coller HA, Sang L, Roberts JM. A new description of cellular quiescence. PLoS Biol 2006;4:e83. doi:10.1371/journal.pbio.0040083.

[38] Dembinski JL, Krauss S. Characterization and functional analysis of a slow cycling stem cell-like subpopulation in pancreas adenocarcinoma. Clin Exp Metastasis 2009;26:611-23. doi:10.1007/s10585-009-9260-0.

[39] Moore N, Lyle S. Quiescent, slow-cycling stem cell populations in cancer: a review of the evidence and discussion of significance. J Oncol 2011;2011. doi:10.1155/2011/396076.

[40] Ciccia A, Elledge SJ. The DNA damage response: making it safe to play with knives. Mol Cell 2010;40:179-204. doi:10.1016/j.molcel.2010.09.019.

[41] Matsuoka S, Huang M, Elledge SJ. Linkage of ATM to cell cycle regulation by the Chk2 protein kinase. Science 1998;282:1893-7.

[42] Jongmans W, Artuso M, Vuillaume M, Brésil H, Jackson SP, Hall J. The role of Ataxia telangiectasia and the DNA-dependent protein kinase in the p53-mediated cellular response to ionising radiation. Oncogene 1996;13:1133-8.

[43] Ahn JY, Schwarz JK, Piwnica-Worms H, Canman CE. Threonine 68 phosphorylation by ataxia telangiectasia mutated is required for efficient activation of Chk2 in response to ionizing radiation. Cancer Res 2000;60:5934-6.

[44] Storch K, Cordes N. Focal Adhesion-Chromatin Linkage Controls Tumor Cell Resistance to Radio- and Chemotherapy. Chemother Res Pract 2012;2012:1-10. doi:10.1155/2012/319287.

[45] Dillon N. Heterochromatin structure and function. Biol Cell 2004;96:631-7. doi:10.1016/j.biolcel.2004.06.003.

[46] Cheung P, Allis CD, Sassone-Corsi P. Signaling to Chromatin through Histone Modifications. Cell 2000;103:263-71. doi:10.1016/S0092-8674(00)00118-5. 